\title{
VACANCY CLUSTERS AT NANOPARTICLE SURFACES
}

\author{
Jun $\mathrm{Xu}^{1}$, J. Moxom ${ }^{1}$, B. Somieski ${ }^{1}$, C. W. White ${ }^{1}$, \\ A. P. Mills ${ }^{2}$, Jr., R. Suzuki ${ }^{3}$, S. Ishibashi ${ }^{3}$, A. Ueda ${ }^{4}$, and D. Henderson ${ }^{4}$ \\ 'Oak Ridge National Laboratory, P. O. Box 2008, Oak Ridge, TN 37831-6142 \\ ${ }^{2}$ Bell Laboratories, Lucent Technologies, 600 Mountain Ave., Murray Hill, NJ 07974 \\ ${ }^{3}$ Electrotechnical Laboratory, 1-1-4 Umezono, Tsukuba, Ibaraki 305, Japan \\ ${ }^{4}$ Physics Department, Fisk University, 1000 17th Ave. North, Nashville, TN 37208
}

Kevwords: nanoparticles, nanosurfaces, vacancy, beam positron lifetime spectroscopy, 2D-Doppler broadening coincidence

\begin{abstract}
We detect vacancy clusters at Au nanoparticle surfaces using a combination of positron lifetime spectroscopy, 1- detector, and 2-detector measurements of Doppler broadening of annihilation radiation. Gold nanoparticles are formed by $\mathrm{MeV}$ implantation of gold ions into $\mathrm{MgO}$ (100) followed by annealing. Clusters of two $\mathrm{Mg}$ and two $\mathrm{O}$ vacancies $\left(\mathrm{v}_{4}\right)$ are attached to the gold nanoparticle surfaces within the projected range $\left(R_{p}\right)$.
\end{abstract}

Nanoparticles are important because they exhibit unique properties, including size effects, which determine colors of luminescence of nanocrystals; quantum mechanical tunneling, which causes "insulators" to lose their ability to isolate; and quantum confinement, which enables conductors to become more resistive. These novel properties will be important for new generations of semiconductor and optical communication devices that are being driven to nanoscale dimensions. Interestingly, the surface-to-bulk ratio is high as materials are reduced to the nanometer scale. Therefore, surface structure can dominate bulk properties, and understanding the surface defects of nanoparticles becomes more urgent. A new tool is needed to characterize nanoparticle surfaces. In this paper, we present advanced positron spectroscopy for revealing clusters of four atomic vacancies in the surfaces of colloidal gold particles embedded in crystalline $\mathrm{MgO}$.

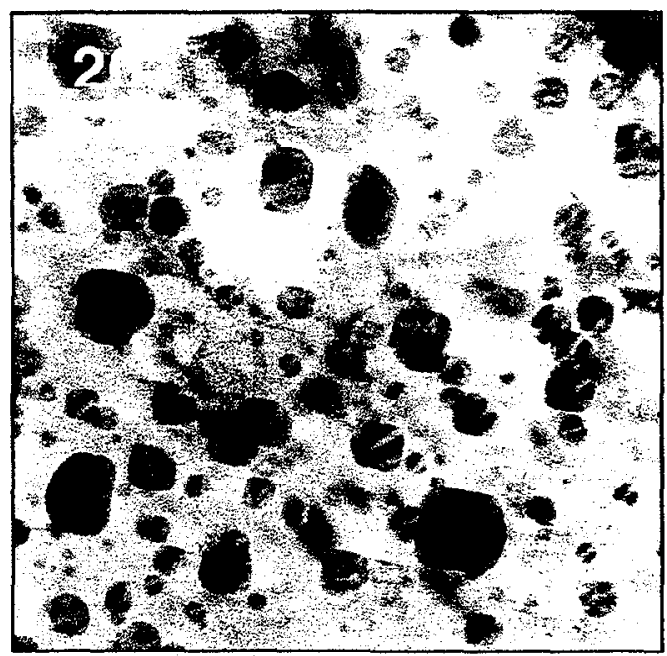

Figure 1 Cross section TEM image of $A u$ nanoparticles embedded in $\mathrm{MgO}$.
Gold nanoparticles are formed in the near surface region of single crystals of $\mathrm{MgO}$ using a combination of ion implantation and thermal annealing.[1] Gold nanoparticles in magnesia are fabricated at the ORNL Surface Modification and Characterization Facility by ion implantation (1.1 MeV gold ions at doses of $1,3,6$, and $10 \times 10^{16} \mathrm{Au}$ ions $/ \mathrm{cm}^{2}$ ), followed by sequential annealing. $\mathrm{Au}$ particles are detected in the range of 1.6 4 $\mu \mathrm{m}$ from the surface, as shown in Figure 1. These nanoparticles are not spherical, but approximately cubic or rectangular, which is believed to be due to $\mathrm{MgO}$ properties. The $\mathrm{Au}$ nano particles have sizes that are in $2-18 \mathrm{~nm}$.

Positron lifetime spectra are measured for both a deep $\mathrm{MgO}$ layer where $\mathrm{Au}$ implantation least affects the data and for the Au nanoparticle layer.[2] Both spectra were deconvoluted using Laplace inversion into the probability density functions (pdf) as a function of positron 
lifetime. Figure 2 shows the pdf spectra for $\mathrm{MgO}$ layer (open circles) and $\mathrm{Au}$ nanoparticle layers (solid circles), respectively. Positron lifetime components for the $\mathrm{MgO}$ layer are $(0.22 \pm 0.04) \quad$ ns with $(89 \pm 3 \%)$ contribution ( $\mathrm{v}_{1}$ in Fig. 2) and (0.59 $\pm 0.07)$ ns with $(11 \pm 3 \%)$ contribution, while those for the $\mathrm{Au}$ nano layer are $(0.41 \pm 0.08) \mathrm{ns}$ at $(90 \%)$ and $(1.8 \pm 0.3) \mathrm{ns}$ at $(7 \%)$. The positron lifetimes are calculated for different defects in $\mathrm{MgO}$ using Puska and co-workers' insulator model in which the annihilation rates are determined by the positron density overlapping with the enhanced electron density that is proportional to the atomic polarizability of $\mathrm{MgO}$ [3]. Based on the

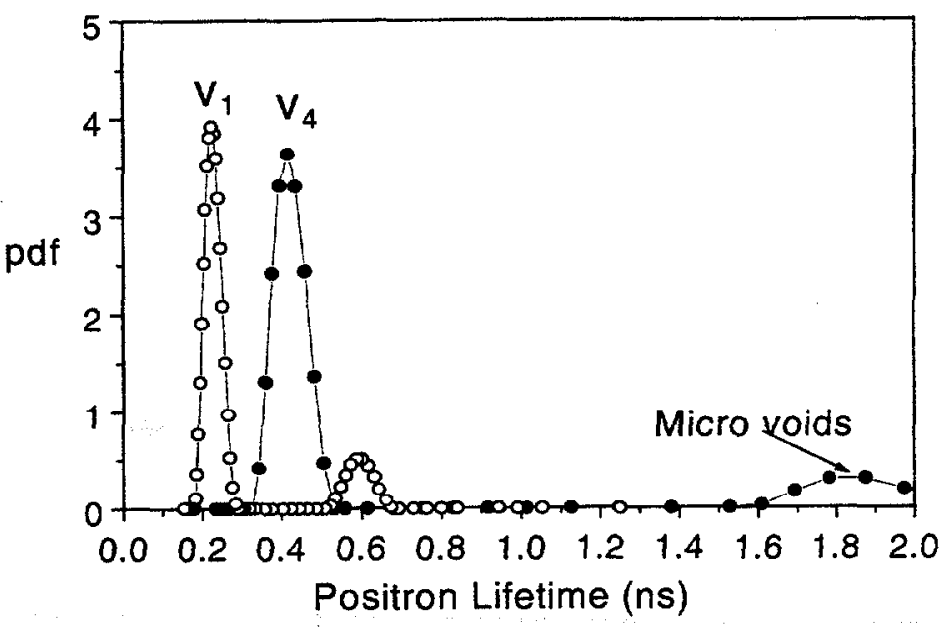

Figure 2. Probability density functions as a function of positron lifetime, resulting from Laplace inversion (CONTIN) of lifetime spectra for $\mathrm{MgO}$ layer (open circles) and $\mathrm{Au}$ nanoparticles embedded in $\mathrm{MgO}$ (solid circles). closeness of the measured lifetimes to the calculated values, the $0.41 \mathrm{~ns}$ measured from $\mathrm{MgO}$ annealed after implantation indicates that the positrons are predominantly trapped in clusters consisting two $\mathrm{Mg}$ vacancies and two $\mathrm{O}$ vacancies. We denote these specific four vacancy clusters as " $\mathrm{v}_{4}$ ". Measurements of Doppler broadening of annihilation radiation were conducted using the variable-energy slow positron beam at ORNL. Figure 3 shows the $S$ parameter measured as a function of positron energy, corresponding to depth shown in the top scale, for $\mathrm{MgO}$ samples that contain Au nanoparticles. For as-grown $\mathrm{MgO}$, the $\mathrm{S}$

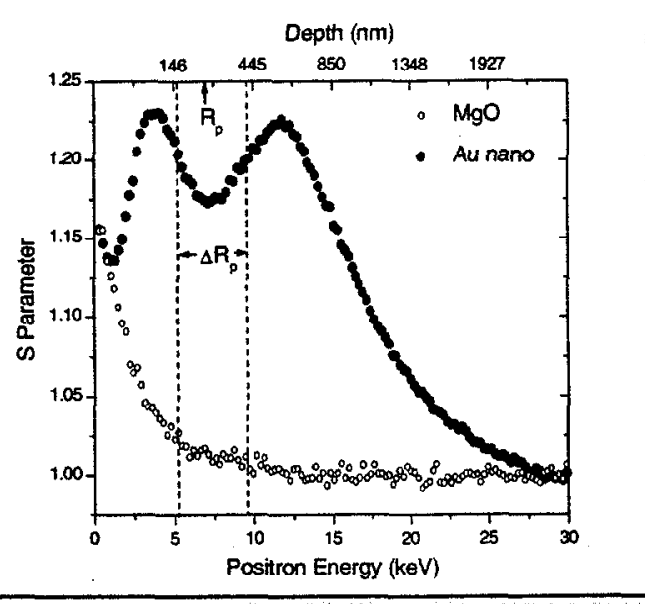
parameter (open circles) is mostly low, except for the near surface imperfections.

After Au nanoparticle formation, the S parameter (solid circles) is greatly increased in the region between the surface and the projected range $\left(R_{p}\right)$. The increased $S$ parameter indicates that a large number of vacancies are produced by ion implantation. The $\mathrm{S}$ parameter also increases for depths greater than $R_{p}$, suggesting that vacancies extend to the region beyond the reach of the $\mathrm{Au}$ ions due to damage by low-mass knock-on ions. It appears that there is a "valley" in the $S$ parameter profile, which is coincident with the Au nanoparticle layer. At this depth, some positrons apparently annihilate with the electrons associated with the Au. We interpret the data as

indicating that $\mathrm{v}_{4}$ clusters are formed on Au nanoparticle surfaces. In this case positrons remain

Figure $3 S$ parameters as a function of positron energy for $\mathrm{MgO}$ samples with (solid circles) and without (open circles) embedded Au nanoparticles. trapped in the $\mathrm{v}_{4}$ sites which apparently yield a lifetime indistinguishable from the undecorated $\mathrm{v}_{4}$ lifetime. However, when the trapped positrons annihilate with near-by-Au electrons, the S parameter is expected to be lower than that of the defected $\mathrm{MgO}$ bulk

To confirm vacancy clusters at nanoparticle surfaces, we have conducted two detector coincidence measurements of Doppler broadening annihilation radiation, a system similar to that developed by Asoka-Kumar and Lynn.[4] Figure 4(left) shows our two dimensional data, while Fig. 4(right) shows the normalized ratio as a function of photon energy for Au nanoparticle (solid circles) and $\mathrm{MgO}$ layers (open circles). In the high-energy range $(<513 \mathrm{keV})$, the spectrum for $\mathrm{Au}$ nanoparticle layer shows two enhanced bands at $514.3 \mathrm{keV}$ and $518.5 \mathrm{keV}$. These bands are 
dramatically different from that of the $\mathrm{MgO}$ layers, where normalized intensity is flat above the silicon. The difference is largely attributed to the surrounding $\mathrm{Au}$ atoms of the defects since the $\mathrm{Au}$ nanoparticle is different for the two environments. To confirm that the difference is due to the $\mathrm{Au}$ atoms, we measure the Doppler broadening spectrum for a pure Au film, shown as the solid line in Fig. 4(right). The Si-normalized spectrum for Au film also shows two main enhanced bands: 514.3 $\mathrm{keV}$ and $518.5 \mathrm{keV}$. This is consistent with the features of $\mathrm{Au}$ films observed in Myler and coworkers' work [5], in which Au annihilation line was also normalized by that of perfect Si. There are a high degree of similarity between the Au films structures and the Au nanoparticle layer. This supports our interpretation that the positrons, trapped at the $\mathrm{v}_{4}$ sites (as we know it from lifetime spectra), annihilate with the electrons associated with Au nanoparticles. Of course, the defects' environment includes the $\mathrm{MgO}$ matrix, which also contributes to the energy deviation spectrum. We conclude that vacancy sites where positrons are trapped are located in the Au nano surfaces.
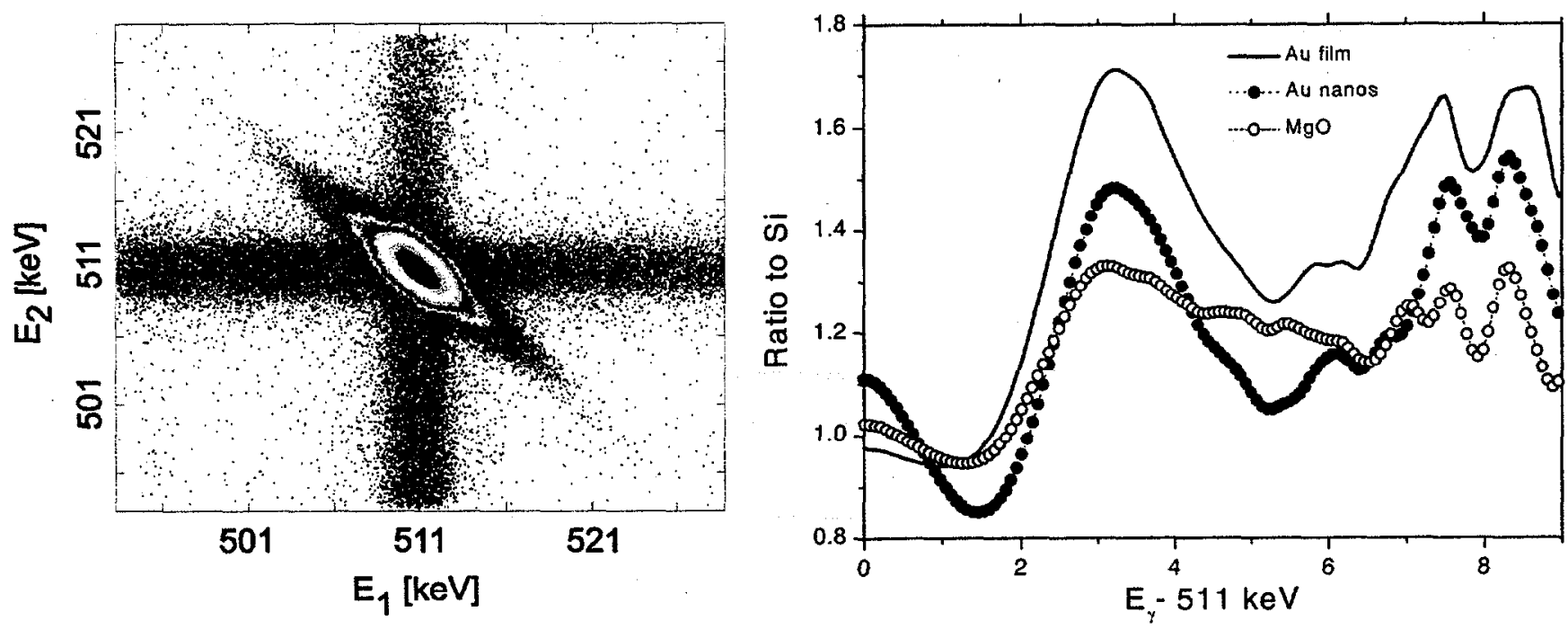

Figure 4 (left) Two-dimensional spectrum of annihilation radiation of positrons injected into a p-Si (100), with $8 \Omega$ $\mathrm{cm}$. The diagonal feature indicates the condition of $E_{1}+E_{2}=1.022 \mathrm{MeV}$; (right) Normalized annihilation lines as a function of photon energy for Au nanoparticle layer (solid circles), $\mathrm{MgO}$ layer (open circles), and Au film (solid line).

In conclusion, we combine both positron lifetime spectroscopy, which tells the existence of clusters of four atomic vacancies in Au nanoparticles embedded in $\mathrm{MgO}$, and 2D-DBAR measurements, which reveals that these clusters are located on the surfaces of Au nanoparticles. The Au implants are not detectable until they form nanoparticles that are associated with vacancy defects. Our study also demonstrates that a positron probe can be used to specify defects of nanosurfaces, which is important as nanoscale fabrication becomes more prevalent.

[1] A. Ueda, R. Mu, Y.S Tung, M. Wu, W.E. Collins, D. O. Henderson, C. W. White, R. A. Zuhr, J. D. Budai, A. Meldrum, P. W. Wang, and X. Li, Nucl. Instru, Meth. Phys. Res. B, 141, 261 (1998).

[2] Jun Xu, A. P. Mills, Jr., A. Ueda, D. Henderson, R. Suzuki, and S. Ishibashi, Phys. Rev. Lett. 83, 4586, (1999).

[3] M. J. Puska, S Makinen, M. Manninen, and R. M. Nieminen, Phys. Rev. B 39, 7666 (1989).

[4] P. Asoka-Kumar, M. Alatalo, V. J. Ghosh, A. C. Kruseman, B. Nielsen, and K. G. Lynn, Phys. Rev. Lett. 77, 2097 (1996).

[5] U. Myler, R. D. Goldberg, A. P. Knights, D. W. Lawther, and P. J. Simpson, Appl. Phys. Lett. 69, 333(1996).

Research sponsored by the Division of Chemical Sciences, Geosciences, and Biosciences, Office of Basic Energy Sciences, U.S. Department of Energy, under Contract DE-ACO5000R22725 with Oak Ridge National Laboratory, managed and operated by UT-Battelle, LLC. 